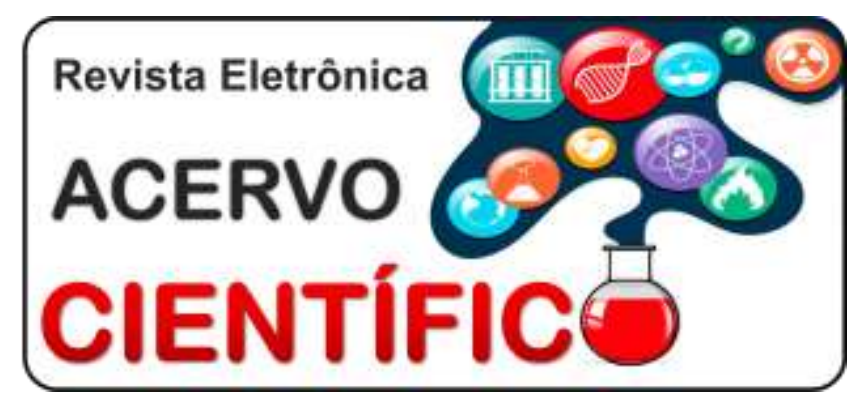

\section{REVISÃO BIBLIOGRÁFICA}

Recebido em: 10/2019

Aceito em: 11/2019

Publicado em: 12/2019

\title{
Injúrias por baixa temperatura em frutos: Um enfoque no uso de tecnologias para retardar as injúrias pós-colheita - Revisão narrativa
}

\author{
Low-temperature fruit injuries: A focus on the use of technologies to delay post-harvest \\ injury - Narrative Review
}

Lesiones de baja temperatura en frutas: un enfoque en el uso de tecnologías para retrasar las lesiones posteriores a la cosecha - Revisión narrativa

Sueisla Lopes Rezende-Silva ${ }^{1}$, Clarice Aparecida Megguer² e Marília Assis dos Santos ${ }^{3}$.

\begin{abstract}
Resumo: Essa revisão aborda acerca dos efeitos e distúrbios fisiológicos provocados pelo frio em frutos tropicais e subtropicais com enfoque no uso de tecnologias para retardar as injúrias pós-colheita. A injúria pelo frio promove distúrbios fisiológicas e bioquímicos em frutos quando expostos a temperaturas abaixo do ponto crítico. Existem vários estudos abordando as injúrias causadas pelo chilling em frutas, entretanto, estudos relatando os efeitos da baixa temperatura com frutos do Cerrado são incipientes. Algumas espécies possuem sensibilidade à refrigeração, podendo ser sujeitas a diversas alterações metabólicas e consequentemente perdas nutricionais e de valor de mercado. Entretanto, existem algumas técnicas que podem atenuar a injúria pelo frio em frutos tropicais e subtropicais. Tecnologias como o uso de aplicativos para smartphone, ultrasom, bem como aplicações de fenilalanina, glicina betaína com finalidade de avaliar e minimizar as lesões por frio, permitem novas perspectivas de uso na pós colheita na avaliação de danos causados por frio.
\end{abstract}

Palavras-chave: Pós-Colheita, Frutos Tropicais e Subtropicais, Frutos do Cerrado.

\begin{abstract}
This review addresses the effects and physiological disturbances caused by cold in tropical and subtropical fruits focusing on the use of technologies to delay postharvest injuries. Cold injury promotes physiological and biochemical disturbances in fruits when exposed to temperatures below the critical point. There are several studies addressing the injuries caused by chilling in fruits, however, studies reporting the effects of low temperature with Cerrado fruits are incipient. Some species have sensitivity to refrigeration and may be subject to various metabolic changes and consequently nutritional and market value losses. However, there are some techniques that can mitigate cold injury in tropical and subtropical fruits. Technologies such as the use of smartphone applications, ultrasound, as well as phenylalanine, glycine betaine applications for the purpose of assessing and minimizing cold injuries, provide new perspectives for postharvest use in assessing cold damage.
\end{abstract}

Keywords: Postharvest, Tropical and Subtropical Fruits, Chilling Injury, Fruits of the Cerrado.

\footnotetext{
${ }^{1}$ Universidade Federal de Goiás (UFG), Unidade Acadêmica Especial de Ciências Biológicas - Regional Jataí, Goiás, Brasil. E-mail: sueislalopes@gmail.com

${ }^{2}$ Instituto Federal de Educação, Ciência e Tecnologia Goiano (IFGoiano), Campus Morrinhos, Goiás, Brasil.

${ }^{3}$ Instituto Federal de Educação, Ciência e Tecnologia de Rondônia, Campus Jaru, Rondônia, Brasil.
} 
Resumen: Esta revisión aborda los efectos y las perturbaciones fisiológicas causadas por el frío en las frutas tropicales y subtropicales, centrándose en el uso de tecnologías para retrasar las lesiones poscosecha. La lesión por frío promueve trastornos fisiológicos y bioquímicos en las frutas cuando se expone a temperaturas por debajo del punto crítico. Hay varios estudios que abordan las lesiones causadas por el enfriamiento de las frutas, sin embargo, los estudios que informan los efectos de la baja temperatura con las frutas Cerrado son incipientes. Algunas especies tienen sensibilidad a la refrigeración y pueden estar sujetas a diversos cambios metabólicos y, en consecuencia, a pérdidas nutricionales y de valor de mercado. Sin embargo, existen algunas técnicas que pueden mitigar las lesiones por frío en las frutas tropicales y subtropicales. Tecnologías como el uso de aplicaciones para teléfonos inteligentes, ultrasonido, así como aplicaciones de fenilalanina, glicina betaína con el fin de evaluar y minimizar las lesiones por frío, proporcionan nuevas perspectivas para el uso posterior a la cosecha en la evaluación del daño por frío.

Palabras clave: Post cosecha, Frutas tropicales y subtropicales, Frutos del Cerrado.

\section{INTRODUÇÃO}

A temperatura exerce influência na conservação de frutos após a colheita (AGHDAM MS e BODBODAK $S$, 2013). É apontada como um dos principais fatores de perda pós-colheita, podendo promover aumento na taxa respiratória e consequentemente antecipação do amadurecimento (CHITARRA MIF e CHITARRA AB, 2005; DREHMER AM et al., 2008). Os distúrbios causados pelo frio em frutos, ocorrem quando os mesmos, são expostos, a temperaturas abaixo do ponto crítico, com efeitos mais intensos em frutos tropicais e subtropicais, tendo como primeira manifestação mudanças estruturais nos cloroplastos (ALMEIDA RF et al., 2005).

Geralmente as injúrias por frio em frutos tropicais e subtropicais são evidenciadas após um período de exposição a temperaturas de refrigeração abaixo de 10 a $15^{\circ} \mathrm{C}$ (graus celsius), mas acima de seus pontos de congelamento (WANG CY, 2010). Frutas quando refrigeradas podem ser suscetíveis à deterioração, entretanto, os sintomas de injúrias "chilling" são exteriorizados quando os frutos ou vegetais são removidos para temperaturas mais elevadas (WANG AL, 2006). Vários estudos abordam os efeitos da temperatura em frutas tropicais e subtropicais, como por exemplo, com frutas de bananeira (OLIVEIRA JAA et al., 2016); abacaxizeiro (SCHAEFFER WA et al., 2019), mamoeiro (ROCHA RHC et al., 2005; ALMEIDA RF et al., 2005; PAN Y et al., 2019); abacateiro (DONADON JR, 2009) e ameixa (HEINZEN AS et al., 2017). Entretanto, existem poucos estudos relatando os efeitos da temperatura em espécies frutíferas do Cerrado.

O Cerrado possuí alta diversidade florística, com várias espécies endêmicas, no entanto, nos últimos anos tem perdido sua biodiversidade devido a intensa ação antrópica (MYERS NRA et al., 2000; MACHADO RB et al., 2004; KLINK CA 2005; SOLÓRZANO A et al., 2012). As frutíferas do Cerrado possuem sabores especiais e alto valor nutricional, elevados teores de açúcares, proteínas, vitaminas, minerais e fibras, o que confere aceitação popular (CAMPOS et al., 2012; LEAO-ARAUJO EF et al., 2019) e potencial uso no cenário agroindustrial brasileiro (REIS AF e SCHIMIELE M, 2019). Desse forma, o conhecimento sobre o comportamento fisiológico pós-colheita de frutos de espécies nativas do Cerrado, podem fornecer informações valiosas sobre as condições ideias de armazenamento, afim de permitir uma maior conservação pós-colheita (CALBO MER et al., 1990), visto que, estes frutos apresentam uma grande perecibilidade (REIS AF e SCHIMIELE M, 2019).

Recentemente Reis AF e Schmiele M (2019), relataram as principais características morfológicas, nutricionais e compostos bioativos dos seguintes frutos do Cerrado: pequi, bocaiuva, mangaba, cagaita, baru, murici, mama-cadela, buriti, araticum e guabiroba, demostrando características distintas entre eles e o potencial uso no processamento de alimentos. LEAO-ARAUJO EF et al., (2019) estudaram a fenologia vegetativa e reprodutiva de plantas de gabiroba (Campomanesia adamantium) na região do Cerrado e relacionam a interferência climáticas, tais como a temperatura, nesses eventos biológicos.

Apesar dos significativos efeitos da elevada temperatura nas perdas pós-colheita, objetiva-se com essa revisão discutir sobre os efeitos e distúrbios fisiológicos provocados pela baixa temperatura em frutos tropicais e subtropicais com enfoque em abordar tecnologias a fim de retardar essas injúrias. 


\section{REVISÃO BIBLIOGRÁFICA}

\section{Temperatura e sensibilidade de frutos a injúrias por frio}

A refrigeração é uma técnica muito utilizada, no propósito de promover a manutenção e a qualidade, bem como aumentar a vida pós-colheita de frutos e hortaliças (CHITARRA MIF e CHITARRA AB 2005). Uma das vantagens em utilizar a redução da temperatura no armazenamento de frutos é que ocorre diminuição do metabolismo, retardo no amadurecimento e senescência (WANG CY, 1993). Algumas espécies são sensíveis à a baixa temperatura, sendo sujeitas a alterações fisiológicas e bioquímicas mais intensas, bem como disfunções celulares, como aumento na síntese de etileno com aumento das taxas respiratórias, aumento da energia de ativação e da permeabilidade e redução da fotossíntese (WANG CY, 2010).

Um dos principais danos provocados em frutos pelo frio são as mudanças na estrutura e composição da membrana. Podendo acarretar perda de controle da permeabilidade e mau funcionamento metabólico, caracterizando estresse oxidativo (Figura 1), quando a atividade enzimática não é suficiente para remover as espécies reativas de oxigênio (BEN-AMOR M et al., 2002). Wanying Ge et al., (2019) relatam que o armazenamento de pimentões a $4{ }^{\circ} \mathrm{C}$ por 25 dias levou ao aumento da fluidez da membrana, induzindo a desestabilização e rompimento das membranas celulares, o que desencadeou sintomas graves de injúrias.

Segundo Chitarra MIF e Chitarra AB (2005) e Sevillano L et al., (2009) os danos causados por baixa temperatura em frutos podem são classificados em: depressões superficiais; colapso interno; colapso do miolo, miolo marron; lanosidade; escaldadura; oleocelose e coração negro (Figura 1). Como efeitos adversos da baixa temperatura em frutos, podem ocorrer desestabilização da membrana resultando na produção de espécies reativas de oxigênio, o que desencadeia estresse oxidativo em frutas sensíveis (Figura 1) (AGHDAM MS e BODBODAK S, 2013).

Figura 1. Cascata de eventos provocados pelo estresse ocasionado pelo frio em frutos.

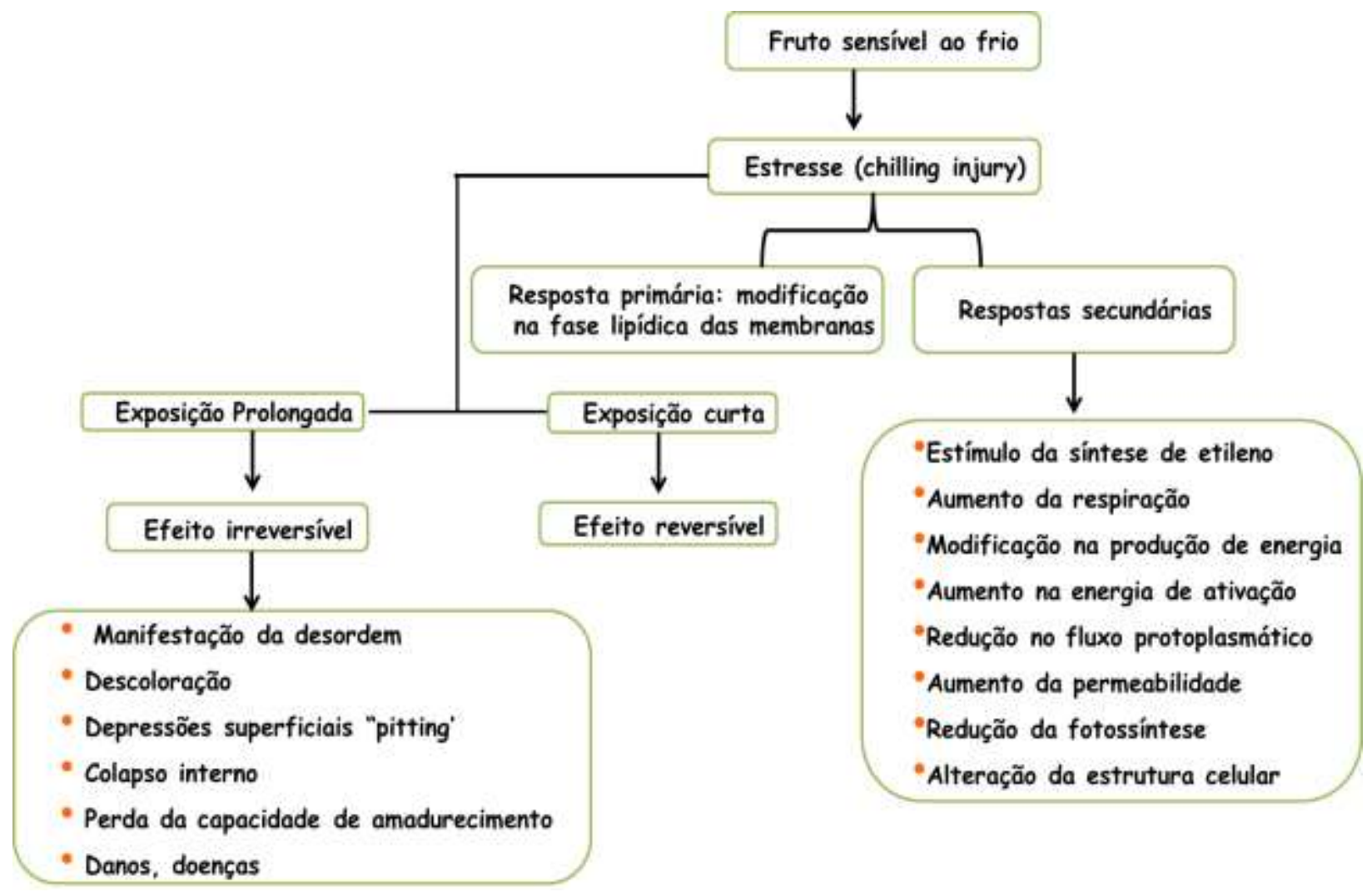

Fonte: Rezende-Silva SL et al., 2019. Adaptado de Chitarra MIF e Chitarra AB, 2005. 
O conhecimento da temperatura de armazenamento adequado para cada espécie é uma importante estratégia a fim de manter uma maior vida pós-colheita de frutos. CHONGCHATUPON AU et al., (2013) estudaram o grau de dano pelo frio em frutos de manga (Mangifera indica) armazenadas a $4{ }^{\circ} \mathrm{C}$ e $12{ }^{\circ} \mathrm{C}$ e observaram que a temperatura de $4 \stackrel{\circ}{\circ}$ promoveu injúria nos frutos, recomendando 0 armazenamento a 12 ${ }^{\circ} \mathrm{C}$ para uma maior manutenção de vida após colheita. Já para frutos de Physalis, o armazenados a $4{ }^{\circ} \mathrm{C}$ durante oito dias, indicam uma melhor manutenção das variáveis físico-químicas como $\mathrm{pH}$, sólidos solúveis totais, firmeza e cor (LIMA CS et al., 2013).

Para frutas cítricas como a laranja "Valência" e "Murcott" e lima "Tahiti" o armazenamento sob refrigeração a $1^{\circ} \mathrm{C}$ e $90-95 \%$ UR (umidade relativa), reduz as injúrias pelo frio e pode prolongar a conservação dos frutos sem afetar as características internas (KLUGE RA et al., 2007). Em contrapartida, o araçá-vermelho que apresenta elevada perecibilidade em condições de temperatura ambiente, é, portanto, recomendado o imediato armazenamento dos frutos a temperaturas próximas de $0 \stackrel{\circ}{ } \mathrm{C}$, visando prolongar a sua conservação (DREHMER AM, 2008). Campos RP et al., (2012) submeteram frutos de gabiroba (Campomanesia sp), uma fruta do Cerrado, a refrigeração, ao 1-MCP (1-Metilciclopropeno) e a atmosfera modificada. Os autores relatam que a conservação pós-colheita de gabiroba é favorecida pelo armazenamento refrigerado a $11^{\circ} \mathrm{C}$ e que o uso de 1 -MCP e da atmosfera modificada prolongam a vida útil dos frutos tanto em armazenamento ambiente, quanto em refrigerado. Para frutos de cagaita, também uma espécie frutífera do Cerrado, o armazenamento a $15{ }^{\circ} \mathrm{C}$, mostrou-se o eficiente para a conservação dos frutos (CALBO MER et al., 1990).

\section{Uso de tecnologias para retardar as injúrias pós-colheita em frutos}

O frio promove distúrbios fisiológicos e bioquímicos em frutos, tecnologias têm sido estudadas a fim de obter técnicas eficientes que atenue o estresse causado pela baixa temperatura. Segundo Wang CY (2010) tratamentos térmicos, aquecimento intermitente, o armazenamento em atmosfera controlada, tratamentos com cálcio, aplicações com etileno, ácido abscísico, poliaminas, jasmonato de metila, ácido salicílico, glicina betaína, por exemplo, consistem em métodos utilizados com a finalidade de atenuar os danos causados pelo frio em frutos. Entretanto, Kluge RA et al., (2007) relatam que nenhum método é capaz de evitar completamente os danos de frio causados pelo armazenamento refrigerado, e sugere que como método básico de controle, os frutos sejam armazenados em temperaturas adequadas para cada espécie. Nesse sentido a ampliação do conhecimento sobre métodos adequados para os diferentes frutos com uso na agroindústria são extremamente relevantes.

Recentemente, Pan $Y$ et al., (2019) estudaram o efeito da glicina betaína em frutos de mamão (Carica papaya L.), tratados com $15 \mathrm{mmol} / \mathrm{L}$ (milimol por litro) de glicina betaína e refrigerados a $6^{\circ} \mathrm{C}$ por 40 dias, os resultados mostraram que o tratamento com glicina betaína reduziu os danos da injúria por frio e proporcionou manutenção da integridade da membrana. A glicina betaína exerce importante papel no ajuste osmótico em células de plantas submetidas a diversos estresses abióticos (MONTEIRO JG et al., 2014).

Estudos realizados por Li P et al., (2014) avaliando os efeitos do ácido oxálico sobre frutos de manga (Mangifera indica L. cv. Zill) quando mergulhados em $5 \mathrm{mM}$ de solução de ácido oxálico em 10 min a $25^{\circ} \mathrm{C}$ e, em seguida, armazenados a baixa temperatura $\left(10 \pm 0,5^{\circ} \mathrm{C}\right)$ durante 49 dias, depois transferidas para 25 ${ }^{\circ} \mathrm{C}$ durante 4 dias, mostram que a aplicação de pré-armazenamento de ácido oxálico inibiu o desenvolvimento de dano pelo frio e que esse efeito do ácido oxálico pode contribuir para melhorar a tolerância a refrigeração minimizando os danos.

Wills RB et al., (2014) estudaram o transporte de bananas durante cinco dias à longa distância, e observaram a relação entre temperatura e etileno, relatando que bananas transportadas no verão com temperatura média de $25^{\circ} \mathrm{C}$ não necessitam de refrigeração se o nível de etileno não exceder $0,58 \mathrm{~mL} \mathrm{~L}^{-1}$, enquanto o transporte no inverno sob temperatura média de $14{ }^{\circ} \mathrm{C}$ poderia se manter níveis baixos de etileno ou reduzir a ação do etileno, sugerindo que o transporte refrigerado de bananas podem ser minimizados ou eliminados. 
O extravasamento de eletrólitos e conteúdo de malonaldeído são indicadores de danos na membrana celular causada por injúria pelo frio em frutos. $O$ armazenamento de frutos de pepino sob baixa e média umidade relativa a uma temperatura de $5 \stackrel{\circ}{\circ}$ promoveu o aumento significativo da taxa de extravasamentos e do conteúdo de malonaldeído, enquanto a alta umidade relativa suprimiu a perda de água e minimizou os sintomas de injúrias por frio (FAHMY K e NAKANO K, 2013).

Para o controle de danos por frio em frutos de lima ácida, a aplicação de carbonato de sódio mostra-se efetiva, seguindo dos tratamentos térmicos, nas faixas de 48 a $54^{\circ} \mathrm{C}$, o que permite concluir que o uso de termoterapia previne o aparecimento de injúrias por frio nesses frutos (NASCIMENTO LM et al., 2013).

A aplicação de óxido nítrico em manga (Mangifera indica L. cv. Orgulho Kensington) após a colheita minimizou as injúrias por frio, com atraso no desenvolvimento da cor, amolecimento e amadurecimento, bem como manutenção da qualidade, através da redução da produção de etileno e respiração durante o amadurecimento (ZAHARAH SS e SINGH Z, 2011).

A utilização do ácido 2,4-diclorofenoxiacético (2,4-D) em mangas injuriadas pelo frio também se mostrou um método eficiente para regular a resistência à refrigeração, por alterar o equilíbrio dos níveis de hormônios endógenos (WANG B et al., 2008). O pré-tratamento com ácido salicílico minimizou danos pelo frio em frutos de pêssegos durante $o$ armazenamento refrigerado pela capacidade de induzir proteínas de choque e sistema antioxidante (KHADEMI O e ERSHADI A, 2013; WANG AL et al., 2006). Efeitos similares usando o ácido salicílico foi observado para estudos com tomates (AGHDAM MS et al., 2012); milho (JANDA T et al., 2013); e damasco (YUAN-YUAN H et al., 2013). Os tratamentos ácido salicílico, ultrassom e a combinação com esses dois tratamentos, diminuíram o dano pelo frio em frutos de bananeira (KHADEMI O et al., 2019).

A aplicação de umidificadores ultrassônicos minimiza as perdas pós colheitas em frutas e vegetais (FABBRI S et al., 2018). A atmosfera controlada, em combinação com temperatura de armazenamento ideal tem sido relatada a prolongar a vida útil de armazenamento e manter a qualidade de frutos (SINGH Z e ZAHARAH SS, 2013; BODBODAK S e MOSHFEGHIFAR, 2016). Nesse sentido, o revestimento de alimentos pode prolongar a vida últil de frutos pós colhidos.

Lio $\mathrm{J}$ et al., (2019) estudaram os efeitos da fibroína na tolerância ao frio em bananas após 4 dias de armazenamento a $6{ }^{\circ} \mathrm{C}$ e relatam que a aplicação de $1 \mathrm{~g} \mathrm{~L}^{-1}$ de fibroína reduziu efetivamente os sintomas de agressão ao frio, sendo uma alternativa viável para manter a integridade da membrana celular, pois aumenta os teores de glicose, frutose e sacarose dos frutos. Os açúcares posseum importante papel na resposta ao frio em frutos de banana na aclimatação (AGOPIAN RG et al., 2011). Os efeitos da baixa temperatura podem ser atenuados por meio do desenvolvimento de cultivares resistentes às baixas temperaturas (ASSMAN AP et al., 2008).

Recentemente Álvarez-Bermejo JA et al,. (2019) relatam sobre o uso de aplicativo para smartphone, como finalidade de avaliar lesões por frio em abobrinha e sugerem que sua aplicação permite novas perspectivas no campo da avaliação de danos causados pelo frio para frutas e legumes em geral. Outra técnica recetemente publicada é o uso da aplicação exógena do aminoácido fenilalanina em frutos. Segundo Aghdam MS et al., (2019) a aplicação de fenilalanina em frutos de tomate durante o armazenamento refrigerado, promoveu maior tolerância ao frio, com possíveis ações no metabolismo eliminado espécies reativas de oxigênio com consequente minimização do estresse oxidativo.

\section{CONSIDERAÇÕES FINAIS}

Nos últimos anos tem se intensificado os estudos sobre os distúrbios fisiológicos provocados pelo frio em frutos tropicais e subtropicais, entretanto, ainda são escassos trabalhos desta natureza relacionados aos frutos do Cerrado com potencial de uso na agroindústria alimentícia. As tecnologias utilizadas para retardar as injúrias pelo frio em frutos tem se mostrado eficientes, possuindo uma especificidade para cada espécie e condições de armazenamento. Estudos têm indicado que o etileno e o estresse oxidativo podem estar envolvidos no processo de indução das injúrias, neste contexto análises multidisciplinar envolvendo desde a expressão gênica, atividade enzimática, parâmetros morfológicos e físico-químicos de pós-colheita, podem 
ser utilizados para se determinar o envolvimento do etileno no mecanismo de indução das injúrias de frio. Tecnologias como o uso de aplicativos para smartphone, ultrasom, bem como aplicações de fenilalanina, glicina betaína com finalidade de avaliar e minimizar as lesões por frio, permitem novas perspectivas de uso na pós colheita na avaliação de danos causados por frio.

\section{REFERÊNCIAS}

1. AGHDAM MS. et al. Alleviation of postharvest chilling injury of tomato fruit by salicylic acid treatment, Journal of Food Science and Technology. 2012; 14: 1-6.

2. AGHDAM MS, BODBODAK S. Postharvest Heat Treatment for Mitigation of Chilling Injury in Fruits and Vegetables. Food Bioprocess Technology, 2013, v. 3.

3. AGHDAM MS, et al. Exogenous phenylalanine application promotes chilling tolerance in tomato fruits during cold storage by ensuring supply of NADPH for activation of ROS scavenging systems. Scientia Horticulturae, 2019. v. 246, p. 818-825.

4. AGOPIAN RGD, et al. Low temperature induced changes in activity and protein levels of the enzymes associated to conversion of starch to sucrose in banana fruit. Postharvest Biology and Technology. 2011, 62 pp. 133-140.

5. ALMEIDA RF, et al. Injúria pelo frio em frutos de mamoeiro (Carica papaya L) cv "Golden". Revista Brasileira de Fruticultura, 2005. v. 27, n.1, p. 17-20.

6. ASSMAN AP, et al. Tolerância de frutos de pessegueiro a geadas. Revista Brasileira de Fruticultura, 2008. v.30, p. 1030-1035.

7. ÁLVAREZ-BERMEJO JA, et al. Development of a smartphone application for assessment of chilling injuries in zucchini. Biosystems Engineering, 2019; 181:114-127.

8. BEN-AMOR M, et al. Inhibition of ethylene biosynthesis by antisense ACC oxidase RNA prevents chilling injury in Charentais cantaloupe melos. Plant, Cell e Environment, 2002. v. 22, n.12, p. 1579-1586.

9. CALBO MER, LIMA CAG. Fisiologia pós-colheita de frutos de cagaita. Revista Brasileira de Fisiologia Vegetal, 1990. v. 2, n. 1, p. 15-18.

10. CAMILLO MF, BENDER RJ. Efeitos de danos pós-colheita por impacto e compressão na qualidade de ameixas. RAMVI, Getúlio Vargas, 2015. v. 02, n. 03.

11. CAMPOS RP, et al. Conservação pós-colheita de guavira (Campomanesia sp). Revista Brasileira de Fruticultura, 2012. v. 34 n.1.

12. CHITARRA MIF, CHITARRA AB. Pós-colheita de frutas e hortaliças: fisiologia e manuseio. Lavras: UFLA, 2005. $785 \mathrm{p}$.

13. CHONGCHATUPON AU, et al. Chilling injury in mango (Mangifera indica) fruit peel: Relationship with ascorbic acid concentrations and antioxidant enzyme activities. Postharvest Biology and Technology, 2013. v. 86, p. 409-417.

14. DONADON JR. Distúrbio fisiológico provocado pelo frio e prevenção com tratamentos térmicos em abacates. Doutor em Agronomia. Faculdade de Ciências Agrárias e Veterinárias, Unesp, Jaboticabal, Brasil, 2009.

15. DREHMER AM, AMARANTE CVT. Conservação pós-colheita de frutos de araçá-vermelho em função do estádio de maturação e temperatura de armazenamento. Revista Brasileira de Fruticultura, 2008.v. 30 n.2.

16. FAHMY K; NAKANO K. Influence of relative humidity on development of chilling injury of cucumber fruits during low temperature storage. Asia Pacific Journal of Sustainable Agriculture Food and Energy (APJSAFE) 2013. v. 1. n 1, p. 1-5.

17. FAO. Técnicas de Manejo Poscosecha a Pequeña Escala: Manual para los Productos Hortofrutícolas (4ª Edición) Control de la temperatura y la humedad relativa. Disponível em: http://www.fao.org/inpho_archive/content/documents/vlibrary/ae075s/ae075s00.htm\#table of contentes. Acessado em 02/12/2013.

18. HEINZEN AS, et al. Qualidade pós-colheita de ameixas 'laetitia' em função do estádio de maturação e temperatura de armazenamento. Revista da Jornada de Pós-Graduação e Pesquisa-congrega urcamp. 2017, 2405-2417.

19. JANDA T, SZALAI G, TARI I, PÁLDI E. Hydroponic treatment with salicylic acid decreases the effects of chilling injury in maize (Zea mays L.) plants. Planta, 2013. v. 08, n. 2, p175-180.

20. KHADEMI O, et al. Effects of salicylic acid and ultrasound treatments on chilling injury control and quality preservation in banana fruit during cold storage. Scientia Horticulturae, 2019. v. 249. p. 334-339.

21. KHADEMI Z, ERSHADI A. Postharvest Application of Salicylic Acid Improves Storability of Peach (Prunus persica cv. Elberta) Fruits. International Journal of Agriculture and Crop Sciences, London, v. 5, n. 6, p. 651-655, 2013.

22. KLINK CA, MACHADO RBA. Conservação do Cerrado brasileiro. Megadiversidade, 2005. 1(1), 147-155.

23. KLUGE RA, et al. Danos de frio e qualidade de frutas cítricas tratadas termicamente e armazenadas sob refrigeração. Revista Brasileira de Fruticultura, 2007. v.29 n. 2.

24. LEÃO-ARAÚJO ÉF, et al. Phenology of Campomanesia adamantium (Cambess.) O. Berg in Brazilian Cerrado. Revista Brasileira de Fruticultura, 2019. 41(2), e-121.

25. LI P, et al. Pre-storage application of oxalic acid alleviates chilling injury in mango fruit by modulating proline metabolism and energy status under chilling stress. Food Chemistry, 2014 v. 142, n. 1. p, 72-78.

26. LIMA CSM, et al. Qualidade pós-colheita de Physalis sob temperatura ambiente e refrigeração. Revista Ceres, 2013. v.60 n.3.

27. LIU J, et al. Fibroin treatment inhibits chilling injury of banana fruit via energy regulation. Scince Horticuture, 2019. 248, pp. 8-13. 
28. MACHADO RB, et al. Estimativas de perda da área do Cerrado brasileiro. Conservation International do Brasil, Brasília, 2004.

29. MONTEIRO JG, et al. Crescimento e conteúdo de prolina em plântulas de guandu submetidas a estresse osmótico e à putrescina exógena. Pesquisa Agropecuária Brasileira, 2014. v. 49, n.1, p. 18-25.

30. MYERS NRA, et al. Biodiversity hotspots for conservation priorities. Nature, 2000. 403, 853-858.

31. NASCIMENTO LM, SANTOS PC DOS. Controle de doenças fúngicas e de danos por frio em pós-colheita de lima ácida Tahiti. Arquivo Instituto de Biologia, 2013. v. 80 n.2.

32. NOVAS N. et al. Development of a smartphone application for assessment of chilling injuries in zucchini. Biosystems Engineering, 2019. p. 114-127, v. 181.

33. OLIVEIRA JAA. et al. Tolerância ao frio de frutos de diferentes cultivares de bananeiras. Revista Caatinga, 2016. 29(3), 629-641.

34. PAIVA A et al. Oranian da Chilling e Freesing - Injúrias causadas por baixas temperaturas em frutas tropicais. Universidade de São Paulo -USP, 2011.

35. PALMER AC, et al. Injúria pelo frio na qualidade pós-colheita de mangas CV. Revista Brasileira de Fruticultura. Jaboticabal - SP. 2011, v. 255-260.

36. PAN Y, et al. Effect of glycine betaine on chilling injury in relation to energy metabolism in papaya fruit during cold storage. Food Scince Nutrition, 2019. 7:1123 - 1130.

37. REIS AF, SCHMIELE M. Características e potencialidades dos frutos do Cerrado na indústria de alimentos. Brazilian Journal of Food Technology, v. 22, 2019.

38. ROCHA RHC. Qualidade pós-colheita do mamão formosa armazenado sob refrigeração. Revista Brasileira de Fruticultura, 2005. 27(3), 386-389.

39. FABBRI S, et al. Improving environmental performance of post-harvest supply chains of fruits and vegetables in Europe: potential contribution from ultrasonic humidification. Journal of Cleaner Production. 2018. 182 pp. 16-26.

40. SCHAEFFER WA, et al. Conservação de cubos de abacaxi submetidos a diferentes embalagens em refrigeração. Anuário Pesquisa e Extensão Unoesc São Miguel do Oeste, 2019. 4, e21091-e21091.

41. SEVILLANO L, et al. Physiological, hormonal and molecular mechanisms regulating chilling injury in horticultural species. Postharvest technologies applied to reduce its impact. Journal Science Food Agriculture, 2009. 89, p. 555573.

42. SINGH Z, ZAHARAH SS. Controlled atmosphere storage of mango fruit - An overview. Acta Horticulturae, 2013. v. 992, p. 481-92.

43. SOUTO RF, et al. Conservação pós-colheita de abacaxi' Pérola' colhido no estádio de maturação pintado associando-se refrigeração e atmosfera modificada. Revista Brasileira de Fruticultura, 2004. 24-28.

44. SOLÓRZANO A, et al. Perfil florístico e estrutural do componente lenhoso em seis áreas de cerradão ao longo do bioma Cerrado. Acta Botanica Brasilica, 2012. 26, (2).

45. WANG AL, et al. Salicylic acid pretreatment alleviates chilling injury and affects the antioxidant system and heat shock proteins of peaches during cold storage. Postharvest Biology and Technology, 2006. v. 41, n. 3, p. $244-251$.

46. WANG B, et al. Reduced chilling injury in mango fruit by 2,4-dichlorophenoxyacetic acid and the antioxidant response. Postharvest Biology and Technology, 2008. v. 48, n. 2, p. 172-181

47. WANG, C.Y. Alleviation of Chilling Injury in Tropical and Subtropical Fruits. Acta Horticutural, 2010. v. 864.

48. WANG, C.Y. Approaches to reduce chilling injury of fruits and vegetables. Horticultural Review, Leriven, 1993. v. 15, p. 63-95.

49. WANYING GE, et al. Insights into the metabolism of membrane lipid fatty acids associated with chilling injury in post-harvest bell peppers. Food Chemistry, 2019. v. 295, p. 26-35.

50. WILLS RBH. Reduction of energy usage during storage and transport of bananas by management of exogenous ethylene levels. Postharvest Biology and Technology, 2014. v.89, p.7-10.

51. YUAN-YUAN H, et al. Effect of Salicylic Acid Treatment on Chilling Injury and the Metabolism of Reactive Oxygen Species in Apricot Fruit Stored at Low Temperature. Food Science, 2013. v. 34, n. 9, p. 277-281.

52. ZAHARAH, S.S; SINGH, Z. Postharvest nitric oxide fumigation alleviates chilling injury, delays fruit ripening and maintains quality in cold-stored 'Kensington Pride' mango. Postharvest Biology and Technology, 2011. v. 60, n. 3, p. 202-210. 